\title{
Plant-derived terpenes: A feedstock for specialty biofuels
}

Ritesh Mewalal ${ }^{1}$, Durgesh K. Rai ${ }^{2}$, David Kainer ${ }^{3}$, Feng Chen ${ }^{4}$, Carsten Külheim ${ }^{3}$, Gary F. Peter ${ }^{5}$ and Gerald A. Tuskan ${ }^{1 *}$

1Biosciences Division, Oak Ridge National Laboratory, Oak Ridge, Tennessee, 37831, USA

${ }^{2}$ Biology and Soft Matter Division, Oak Ridge National Laboratory, Oak Ridge Tennessee, 37831, USA

${ }^{3}$ Research School of Biology, The Australian National University, Canberra, ACT 2601, Australia

${ }^{4}$ Department of Plant Sciences, University of Tennessee, Knoxville, TN 37996, USA,

${ }^{5}$ School of Forest Resources and Conservation, University of Florida, Gainesville, Florida, 32611, USA

*Correspondence: tuskanga@ornl.gov (G.A. Tuskan).

Keywords: specialty biofuels; Eucalyptus; plant secondary metabolites; systems biology; synthetic biology; terpenes 


\section{Abstract}

Research toward renewable and sustainable energy has identified specific terpenes capable of supplementing or replacing current petroleum-derived fuels. Despite being naturally produced and stored by many plants, there are few examples of commercial recovery of terpenes from plants due to low yields. Plant terpene biosynthesis is regulated at multiple levels, leading to wide variability in terpene content and chemistry. Advances in the plant molecular toolkit, including annotated genomes, high-throughput omics profiling and genome editing, have begun to elucidate plant terpene metabolism, and such information is useful for bioengineering metabolic pathways for specific terpenes. Here, we review the status of terpenes as a specialty biofuel and discuss the potential of plants as a viable agronomic solution for future terpene-derived biofuels.

\section{Energy-rich terpenes as specialty biofuels}

In plants, terpenes (or terpenoids or isoprenoids) are a naturally occurring, chemically diverse set of metabolites associated with developmental physiology as well as mutualistic and antagonistic plant-herbivore and plant-environment interactions (Box 1) [1]. Terpenes, together with aromatic compounds, constitute the essential oils of plants, with the highest concentration usually found in the specialized storage cavities of leaves. Terpenes are hydrocarbons that are classified by the number of isoprene units $\left(\mathrm{C}_{5} \mathrm{H}_{8}\right)$, with the most common being mono-, sesqui-, and di-terpenes $\left(\mathrm{C}_{10}, \mathrm{C}_{15}\right.$, and $\mathrm{C}_{20}$, respectively). Commercially, terpenes have industrial uses as agrichemicals, fragrances, nutraceuticals and pharmaceuticals [2]. From a growing repertoire of $40,000+$ reported structures, specific terpenes have been identified as specialty biofuels (see Glossary) that meet current industrial and chemical requirements, including viscosities, flash points, and freezing points, high energy densities and high volumetric net heats of combustion (NHOC) [3, 4]. Table 1 provides a summary of specific biofuel-related terpenes identified.

Terpenes occur in both cyclic and acyclic forms; however, the cyclic forms have higher energy density and $\mathrm{NHOC}$, and are therefore preferred as feedstocks for biofuels [3]. Despite this, high-density fuels have also been synthesized from acyclic linalool and farnesene via ring closure metathesis $[4,5]$. As a biofuel, terpenes can be used directly 
or blended with existing jet fuel (e.g. Jet-A, JP-5 and JP-8), missile propellant (e.g. JP10), gasoline, or diesel fuels [6-9]. For example, the hydrogenated monoterpenes myrcene and limonene were shown to be suitable additives to diesel [9], and $\beta$-pinene has been used to synthesize high-density biofuels comparable to JP-10 (although the viscosity and freezing point were higher) [10]. Meylemans et al. demonstrated that catalytic dimerization of a-pinene, camphene, limonene and crude turpentine (dominated by a-pinene) produces high-density biofuels comparable to JP-10 [6]. Importantly, Meylemans et al. later showed that blending terpene dimers with jet fuel could improve the NHOC and energy density while simultaneously avoiding the high viscosities associated with $\mathrm{C}_{20}$ molecules [7]. Hellier et al. assessed the performance of twelve different terpenes in compression and spark ignition engines. The authors found that terpenes could serve as standalone fuels or in blends up to $65 \%(\mathrm{w} / \mathrm{w})$ in diesel or gasoline engines [8]. Furthermore, Harvey et al. showed that high-density fuels could be generated from hydrogenated valencene, premnaspirodiene and caryophyllene using a heterogeneous acid catalyst, Nafion SAC-13. These fuels could serve as diesel fuels or additives to jet fuels [11]. Traditionally, oxygenated terpenoids have been disregarded as a fuel source; however, using heterogeneous acid catalysts, Meylemans et al. demonstrated the conversion of oxygenated 1,4-cineole and 1,8-cineole to high-density hydrocarbons, which function as blend-in diesel fuel additives [12]. Several patents have been issued or are pending, covering the efficient conversion and use of terpenes as biofuels (U.S. Patent Number: US8227651B1, US9327279B2, US8975463B1 and US2015/0011810A1) [4, 13-15].

Plant-derived terpenes represent an alternate sustainable source of energy, potentially alleviating fossil fuel dependences and associated effects on atmospheric carbon dioxide and climate change [16]. However, naturally derived terpenes have not been commercially implemented as biofuels due to the low yield of specific terpenes in plants. Over the last decade, plant research has experienced considerable growth in omics technologies (i.e., DNA sequencing, RNA sequencing, metabolic profiling and proteomics) and improvements in genome modification/editing tools such as CRISPRCas9 [17]. These developments, combined with the inherent ability of many plants to synthesize and store terpenes, provide a powerful platform for the commercial-scale 
production of terpenes for future biofuels. In this review, we assess synthetic routes towards producing these compounds and highlight plant-based systems as a viable source of terpenes. We further discuss approaches to understand the terpene biosynthetic machinery, and hypothesize future commercial recovery strategies. As an example, we outline the potential of an agronomic system based on coppiced Eucalyptus plantations as a source for terpene production.

\section{Engineering terpene biosynthesis in non-plant systems}

Thus far, efforts to produce terpenes as a specialty biofuel have been limited to microorganisms. The genetic tractability of microbial systems has led to significant progress in reconstructing metabolic pathways within these organisms for several biofuels, including terpenes [18]. Peralta-Yahya et al. identified bisabolane as a suitable alternative to D2 diesel and showed that its precursor, bisabolene, could be produced at high levels in both Escherichia coli $\left(>0.90 \mathrm{~g} \mathrm{l}^{-1}\right)$ and Saccharomyces cerevisiae $(>0.90$ $\mathrm{g} \mathrm{I}^{-1}$ ) by recombinant expression of the Abies grandis (E)- $\alpha$-bisabolene synthase gene. Bisabolene was then converted to bisabolane via chemical hydrogenation [19]. Kirby et al. identified a novel route within E. coli to synthesize the building blocks of terpenes, isopentenyl pyrophosphate (IPP) and dimethylallyl pyrophosphate (DMAPP), using the pentose sugar, ribulose 5-phosphate (Ru5P) instead of pyruvate and glyceraldehyde 3phosphate. Further co-expression with $A$. grandis bisabolene synthase resulted in an improved metabolic flux toward the synthesis of bisabolene and a yield of $>2.0 \mathrm{mg} \mathrm{g}^{-1}$ dry cell weight [20]. Zhang et al. demonstrated through metabolic engineering of the terpene mevalonate (MVA) precursor pathway in $E$. coli, in combination with optimization of growth and culturing conditions, that sabinene could be synthesized at a concentration of $2.65 \mathrm{~g} \mathrm{l}^{-1}$ [21]. Two recent reports demonstrated that the plasticity of the terpene biosynthetic pathway could be exploited for production of $\beta$-caryophyllene at high levels $\left(1.05 \mathrm{gl}^{-1}\right.$ and $\left.1.52 \mathrm{gl}^{-1}\right)$ in engineered $E$. coli $[22,23]$. With the application of systems biology and synthetic biology, metabolic engineering in microbial systems has further potential for commercializing biofuels, including lower cost and high product yield $[24,25]$. 
While microbial expression platforms are promising, they are not without shortcomings, including cellular toxicity associated with terpenes, accumulated toxic intermediates within the pathway, uncontrolled volatilization of terpenes from the culture media, and low titers [8, 18, 26-28]. For example, Hellier et al. showed that even at low concentrations, geraniol and geranial can be toxic to the heterologous expression system [8]. Enzymes from the terpene biosynthetic pathway also require posttranslational modifications (PTMs) for functionality [29], which are absent in many prokaryotic expression systems and culminates in insoluble protein aggregates. Eukaryotic expression systems (e.g., mammalian cell expression), may address this particular disadvantage; however, the cost of batch fermentation can be high, especially for growth media and culturing conditions. Moreover, the overexpression of foreign genes can lead to an imbalance in the host metabolic pathways in the case of enzymes, and exhaust host resources such as energy and amino acids, which is associated with inhibition of normal growth and stress responses [18]. Microbial expression platforms also do not have storage systems innately found in several plants, which may explain the toxicity associated with accumulation of terpenes within microbial cells. Finally, chemical synthesis may not provide a feasible route for low-cost, high-yield terpenes due to the complexity of the chemical structures [30] and subsequent volatility.

\section{Plant terpenes as a source for specialty biofuels}

Considering that plants naturally produce and store thousands of terpenes, including those with a potential as biofuels (Table 1), the question remains: why have plants not been leveraged as a viable source of these compounds? The foliar tissues of many plants, such as Eucalyptus species, contain large natural variations in terpene content and composition [31]. For example, Eucalyptus polybractea showed approximately 20fold natural variation in foliar terpene content $\left(0.7-13.0 \% \mathrm{DW}^{-1}\right.$ in 170 seedlings), dominated by monoterpenes, specifically 1,8-cineole and smaller amounts of spathulenol (3.9\%), a-pinene (2.3\%), limonene (2.6\%), $\alpha$-terpinyl acetate $(1.6 \%), \beta$ pinene $(1.6 \%)$, myrcene $(1.2 \%)$, sabinene $(1 \%)$, $\alpha$-terpineol $(1 \%)$, caryophellene oxide (1\%) [32]. Gene and pathway perturbation for terpenes in several plants have resulted in anticipated changes in terpene content [33]. Lange et al. showed a consistent increase in monoterpene content in transgenic peppermint (>78\%) grown in greenhouse 
and commercial-scale field trials by using candidate gene perturbation [34]. The ability to synthesize terpenes suggest an appropriate physio-biochemical environment that facilitates the correct enzyme folding, subcellular targeting, and PTMs to synthesize the correct atomic configurations of the terpenes [29, 35]. One way that the phytotoxicity associated with terpenes within the plant cellular environment is avoided is by secretory cells. These cells synthesize toxic metabolites and transport them into specialized storage cavities, including resin ducts in gymnosperms, oil glands in Eucalyptus, and glandular trichomes in Mentha. The availability of several plant genomic resources including Phytozome v11 (http://phytozome.jgi.doe.gov/pz/portal.html) and Plaza (http://bioinformatics.psb.ugent.be/plaza/) will facilitate comparative genomics to adopt knowledge learned from model plants to dedicated biomass crops such as Eucalyptus. These crops have marginal land requirements with little to no overlap with land for food crops and have an all-year-round harvesting potential in the case of short rotation coppice Eucalyptus [36]. The commercial-scale production of terpenes from these crops is potentially carbon-neutral because plants sequester large amounts of above- and below-ground carbon, thereby mitigating greenhouse gas emissions [37].

In the past, commercial-scale production of specific terpenes in plants was overlooked due to the relatively low yields from mature plants grown under non-managed, natural systems. Today, however, commercial scale recovery of plant terpenes occurs from pines, eucalypts, mints, and citrus. In the U.S., pine terpenes and fatty acids present in the wood are recovered as co-products during pulping [38]. The global industry annually recovers about 3 million tonnes of terpenes annually. These hydrocarbons are fractionated into mono/diterpenoids and fatty acids, which are used as renewable chemical feedstocks for a large number of products and compete on price and quality with petroleum-derived compounds. In the first 10 years of growth, planted pine trees with an average of $4 \%$ wood terpene content produce $40 \mathrm{GJ} \mathrm{ha}^{-1} \mathrm{y}^{-1}$ of energy in hydrocarbons in the woody stem (G. Peter, personal communication). In contrast to pines and citrus, eucalypt terpenes are extracted from leaves in short-rotation coppice agroforestry systems. This approach can increase leaf biomass production per unit land area per unit time [39]. Furthermore, the availability of the Eucalyptus genome [40] will facilitate the commercial deployment of genetically improved plant with customized 
terpene production and maximized oil gland capacity [41, 42]. Recent research has explored producing biofuels, including Jet-A1, from lignin biomass via pyrolysis methods. Eucalypts, grown and harvested for biomass, can produce biofuel with low ecological impact, but profitability may be questionable in the short to medium term [43]. The addition of a terpene extraction stage to the pipeline may improve the sustainability of such systems by maximizing the volume and variety of fuel obtained from a single harvest [44]. Engineering eucalypts for maximal production of both lignin and terpene presents a challenge as maximizing one may penalize the other, but an optimal balance may result in greater overall production of high value fuels per $\mathrm{kg}$ of harvested dry weight.

Given that 1) we understand the quantitative and qualitative variation of terpenes in eucalypts well [45, 46], and 2) a commercial coppice system for pharmaceutical terpene production exists [47], we can estimate commercial-scale production of biofuels from specific terpenes. At an oil content of $48 \mathrm{mg} \mathrm{g}^{-1}$ fresh weight of leaves and $94 \% 1,8$ cineole suggests $E$. polybractea is capable of producing commercial scale quantities of Eucalyptus oils which consist of terpenes and aromatics [48]. Wu et al. estimated that mallee eucalypts grown under coppice rotations reach an energy productivity of $206 \mathrm{GJ}$ ha $^{-1} \mathrm{y}^{-1}, 35.5 \%$ of which of is from leaves for a production period [49]. Goodger et al. measured the oil yield produced from $E$. polybractea during a 12-month coppicing rotation, comparing 20 randomly selected saplings out of 1000 , sourced from seeds of 30 open pollinated maternal lines. The individual with the highest oil yield produced 137 $\mathrm{g}$ of total oil from $715.6 \mathrm{~g}$ of leaf (dry weight, DW; $19.1 \%$ oil concentration $\mathrm{DW}^{-1}$ ). At the recommended planting density of 5000 plants $\mathrm{ha}^{-1}$ [50], clones of this elite individual have the potential to produce $686 \mathrm{~kg}$ of essential oil ha ${ }^{-1} \mathrm{y}^{-1}[51]$.

Crude steam-distilled oil from pine stems is made up mostly of a-pinene (75-85\%), with smaller amounts of $\beta$-pinene (0-3\%), camphene (4-15\%), and limonene (5-15\%), and traces of terpinolene and 3-carene [52]. The refining steps of these terpenes include hydrotreatment and fractionation to produce biodiesel. The composition of crude oil extracted by steam-distillation from eucalypt leaves is highly variable both between and within species [45]. For example, current populations of E. polybractea contain between 8-98\% 1,8-cineole (Kainer et al., unpublished results). Other eucalypt species, however, 
are dominated by $\alpha$ - or $\beta$-pinene, which can be used directly as fuels (Table 2). With sufficient selection or genetic engineering, oils from eucalypts can contain over $95 \%$ of usable terpenes, which may be separated by fractionation distillation and dimerized with high efficiency. At $500 \mathrm{~kg} \mathrm{ha}^{-1} \mathrm{y}^{-1}$ useable terpene production, 20 million ha (i.e., the current global area planted with eucalypts) would produce 10 million tons of high-energy jet fuel. According to the US States Energy Information Administration (http://www.eia.gov/), daily global jet fuel consumption in 2012 was 5.381 million barrels - an annual consumption of 181 million tons. Therefore, 20 million ha could provide over $5 \%$ of the fuel as additive, which is less than the current production of bioethanol (225 million tons annually [53].

\section{Challenges in understanding the plant terpene biosynthetic machinery}

Despite the potential economic and environmental benefits of terpenes derived from plants, key biological challenges must be overcome. Not all terpenes are represented in all plant lineages, and individuals of the same species may vary in terpene content [54]. There have also been reports of variations in terpene content in response to biotic stress [55], seasonal changes [56], and endogenous hormone levels [57]. Interestingly, terpene diversity is not paralleled by an equivalent number of encoded enzymes. In fact, a single terpene synthase (TPS) may catalyze the synthesis of multiple terpenes [42, 58]. Perturbations of terpene-related genes have been accompanied by inadvertent pleiotropic changes in plant development and terpene content [33]. Phillips et al.

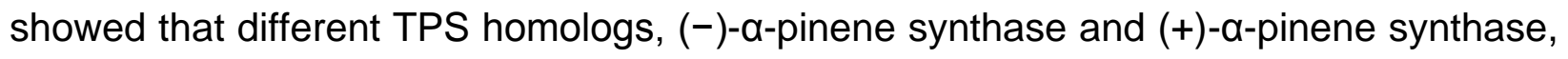
are required to synthesize enantiomers of a-pinene [59], while Peralta-Yahya et al. demonstrated that orthologous TPS vary in their activity to synthesize specific terpenes [19]. Irmisch et al. reported that a single amino acid difference in the active site of Populus trichocarpa PtTPS19 and PtTPS20 produced either ent-kaurene or 16ahydroxy-ent-kaurane in E. coli, highlighting the importance of enzyme specificity at the level of gene variants [60]. These studies demonstrate that terpene biosynthesis is a dynamic process with a complex underlying genetic circuitry, and before the economic and environmental benefit can be recognized, a comprehensive understanding of the biological process is necessary. 


\section{A systems-level understanding of terpene biosynthesis}

In plants, genetic and environmental factors regulate terpene biosynthesis at multiple levels, including transcription, protein accumulation, and posttranscriptional/translational regulation [29, 61]. Understanding the molecular underpinnings of terpene metabolism in plants will benefit from a systems biology approach, where the goal is to quantitatively describe the cellular processes through global modeling of the interactions and dynamics of the molecular components. Systems biology integrates multiple omics datasets through mathematical and probabilistic modeling including correlation networks, graphs, and statistical models, to understand the emergent phenotype of a cell or organism $[62,63]$. While transcriptomic and proteomic studies have catalogued many genes putatively involved in terpene biosynthesis [64-67], these studies in isolation provide only a snapshot of this biological process and do not reflect in vivo conditions. For example, transcriptional profiles of putative homologs of the E. grandis MVA and MEP pathway (Figure 1) revealed that multiple paralogous genes from the MEP pathway exist and are co-expressed across tissues [68]. This phenomenon will complicate metabolic engineering due to genetic redundancy and possible unintended pleiotropic effects. For example, Pasoreck et al. showed broad off-target transcriptomic and metabolomic changes caused by perturbations of squalene biosynthetic genes [69].

Systems biology network outputs are correlations and do not imply causation. However, the approach can identify gene modules, which are groups of highly correlated gene expression patterns, to refine a candidate gene list. Furthermore, positive and negative correlations within networks, across developmental stages, different plant organs (such as whole leaf and oil glands), or from genetically modified plants compared with the wild type, will yield predictive models of pathway regulation and flux [70, 71]. Otero et al. showed in S. cerevisiae that systems biology could be used to rationally select a suite of genes to perturb in order to redirect the carbon flux for improved succinate yield [72]. Metabolic engineering for specific terpenes will have to be tailored, and given the gaps in our understanding of this biological process (Figure 2), systems biology will be instrumental in cataloging the genetic determinants. These determinants include preferentially expressed enzyme variants, transporters for precursors and metabolites, 
signaling and regulators proteins, regulatory RNAs, and others. Furthermore, depending on the initial experimental design, network analysis can reveal gene-gene relationships, feedback loops, pathway flux, spatial-temporal regulation and relationships to different biochemical pathways (which may circumvent unwanted pleiotropy during gene perturbation) to predictively maximize terpene biosynthesis in metabolic engineering strategies.

The system-level understanding of terpene biosynthesis may be extended beyond the traditional molecular dissection to include the physical characterization of storage cavities and terpene-terpene dynamics. Storage cavities contain a complex mixture of nonvolatile terpenes, volatile terpenes, and other secondary metabolites such as phloroglucinol compounds. The phase behavior of the entrapped volatile and nonvolatile terpenes is poorly understood but may have important implications for terpene-terpene interaction and stability, relating the structure of oil glands to their function and understanding the transport of terpenes into the cavity. Scanning electron microscopy revealed modified epidermal cells surrounding the storage cavities [73], while confocal fluorescence microscopy has revealed the spatial organization of cavity metabolites, with the nonvolatile components forming a layer between secretory cells living in the lumen and the volatile terpenes [74]. Based on SEM and transmission electron microscopy analysis, the volatile terpenes in the cavities are thought to pass through modified epidermal cells that cap the oil glands, allowing a gradual loss of essential oils from the oil glands when exposed to vacuum conditions [75]. Neutron scattering techniques, such as small-angle neutron scattering and ultra-small angle neutron scattering, are emerging complementary techniques that can be used to characterize the structural properties of oil glands. The highly penetrating neutrons and the ability to selectively highlight specific regions in a macromolecular complex by deuterium/hydrogen atom exchange is advantageous for studying complex biological systems. These technologies are able to resolve structural details on length scales from $\sim 1 \mathrm{~nm}$ to $100 \mu \mathrm{m}$ [76-78] and have the potential to elucidate the internal structure of oil glands. 


\section{Commercialization potential of terpenes from plants}

To produce terpene-based fuels at commercially competitive scales, gains in terpene yield per hectare could be made by producing elite lines with consistently high foliar terpene content, establishing silviculture techniques, and decreasing terpene loss during harvesting and extraction. A crucial step is to identify species with high foliar content of specific terpenes that grow well on marginal lands with minimal impact on food production and native biodiversity. Padovan et al. (2014) reviewed the dominant terpenes from 1393 species of Myrtaceae, including 648 species of eucalypts (genera of Eucalyptus, Angophora and Corymbia) [45]. Of these eucalypts, fifteen species have oil profiles dominated by $\alpha$-pinene, $\beta$-pinene, or $p$-cymene (Table 2), and most have good abilities to re-sprout after coppicing and are able to grow in the semi-arid sub-tropic climate zones.

A systems-level understanding of terpene biosynthesis forms an umbrella over two foreseeable translational strategies for the development of elite lines: synthetic biology and traditional selective breeding strategies. Synthetic biology in plants will integrate predictive modeling from systems biology with a high-precision genome editing toolkit, including assembly of multigene constructs for gene stacking [79], high-throughput genome modification (CRISPR-Cas9) [9], and spatial-temporal regulation [80] to design metabolic pathways and fluxes for optimized production of specific terpenes. A number of genetic tools are available or being optimized for plant modification which will serve as a foundation for biotechnological engineering applications [81]. Moreover, studies have reported successful Agrobacterium-mediated genetic transformation of many plants, including Eucalyptus species [41, 82]. In addition to affecting terpene content, synthetic design should also target terpene stability and storage capacity in plants. For example, monoterpenes have been reported to exist in nonvolatile glycosylated forms. Therefore, identifying specific glycosyltransferases catalyzing such sugar conjugations to increase terpene stability and water solubility will be important for future biotechnology [83, 84]. Genetic promoter sequences that are specific to the storage organs, such as those reported by Kortbeek et al., will be useful to prevent undesired pleiotropy or phytotoxicity associated with terpenes in non-native cells [85]. 
Eucalyptus oil yield is a complex quantitative trait that is influenced by foliar oil concentration, oil composition, leafy biomass accumulation and leaf morphology [86]. Terpene yield, in particular, displays large variance and high heritability, so selective breeding for these traits is likely to generate significant gains. For example, in a related tea tree, three cycles of phenotypic selection over 20 years resulted in doubling the oil yield from 148 to $300 \mathrm{~kg} \mathrm{ha}^{-1}$ [87]. This process could be accelerated in undomesticated eucalypt species through molecular breeding techniques such as genomic selection [61]. Previous studies reported that the terpene content is limited by the storage gland capacity, which in turn is regulated by the leaf area and thickness [46]. Furthermore, Goodger and Woodrow showed that oil concentration in E. polybractea is tightly correlated with the total volume of secretory oil cavities $\left(r^{2}=0.96\right)$, which in turn is constrained by leaf size and metabolic support requirements [88]. Therefore, improving other traits such as leafy biomass accumulation, crown form, post-coppice regrowth rate, and even herbivory resistance may provide additional paths to higher overall yield. E. polybractea is typically harvested on a 2 year rotation and may be harvested for up to 100 years, but shorter rotation times (e.g., 1 year) result in diminishing returns as the trees are unable to fully recover lignotuber mass between harvests [89]. The development of viable short-rotation genotypes has the potential for very large annualized yield gains in short to medium term.

Intensive silvicultural management will also play an important role in maintaining productivity from elite plant lines. Silviculture considerations include plantation sites, planting density, crop rotation (harvesting), coppicing ability, fertilizer requirements, and pest management. For terpene production in the foliage of Eucalyptus, shorter rotations will be advantageous, so densities can be in the range of $\sim 3000-5000$ trees $^{-1}$ to maximize per ha productivities [50, 90]. Silviculture techniques will have to be established, if they have not been already, for specific plant genera. For example, plantation sites will be dictated by cold tolerance of the crop species. Currently, 50 000 ha of Eucalyptus are planted in California, Hawaii, and Florida [90]; however, expanding this distribution, particularly into the south of the US, will require frost-tolerant species [91]. 
Finally, with current short-rotation coppice practices, a proportion of the essential oil present in Eucalyptus foliage is lost during harvesting, and up to $25 \%$ is left unextracted due to inefficiencies in steam distillation [92]. Improvements in harvesting and distillation efficiency will be important factors for net energy gain. Ongoing studies have demonstrated improvements in extraction of specific terpenes from complex mixtures in plants $[93,94]$.

\section{Concluding remarks}

Plants are attractive systems for the commercial-scale production of specific terpenes due to their inherent ability to synthesize, transport, accumulate, and store these compounds. To leverage plant systems for terpenes requires addressing several challenges (Box 1). A roadmap for the commercial recovery of terpenes synthesized in plant is proposed in Figure 3. Exploiting the existing variation in plant terpene content and using high-throughput omics profiling technology should lead to a fundamental understanding of this biological process and facilitate predictive engineering of plants for specific terpenes using synthetic biology (single gene effects or additive effects of gene stacking to rewire the terpene metabolic pathway), selective breeding approaches, and their combination. Together with well-established silviculture and harvesting practices, terpenes synthesized in planta hold tremendous potential as a sustainable specialty biofuel.

\section{Acknowledgements}

This review is based on work supported by the Department of Energy (DOE) and funding from the LDRD project LOIS ID: 7428 . The contents of this review are solely the responsibility of the authors and do not necessarily represent the official views of the DOE. The authors acknowledge Lee E. Gunter, Aparna Annamraju, Sai Venkatesh Pingali, Hugh M. O'Neill, Daniel Jacobson and Timothy J. Tschaplinski for their critical review of the manuscript. Oak Ridge National Laboratory is managed by UT-Battelle, LLC for the US DOE under Contract Number DE-AC05-000R22725. 


\section{References}

1 Gershenzon, J. and Dudareva, N. (2007) The function of terpene natural products in the natural world. Nat. Chem. Biol. 3, 408-414

2 Kirby, J. and Keasling, J.D. (2009) Biosynthesis of plant isoprenoids: perspectives for microbial engineering. Annu. Rev. Plant Biol. 60, 335-355

3 Filley, J., et al. (2001) Energetics of the 2+ 2 cyclization of limonene. J. Photochem. Photobiol. A: Chem. 139, 17-21

4 Harvey, B.G. United States of America as Represented by the Secretary of the navy, Arlington, VA (US). High density cyclic fuels derived from linear sesquiterpenes, US2015/0011810A1

5 Meylemans, H.A., et al. (2011) Solvent-free conversion of linalool to methylcyclopentadiene dimers: A route to renewable high-density fuels. ChemSusChem 4, 465-469

6 Meylemans, H.A., et al. (2012) Efficient conversion of pure and mixed terpene feedstocks to high density fuels. Fuel $97,560-568$

7 Meylemans, H.A., et al. (2013) Low-temperature properties of renewable high-density fuel blends. Energy Fuels 27, 883-888

8 Hellier, P., et al. (2013) Combustion and emissions characterization of terpenes with a view to their biological production in cyanobacteria. Fuel 111, 670-688

9 Tracy, N.I., et al. (2009) Hydrogenated monoterpenes as diesel fuel additives. Fuel 88, 2238-2240

10 Harvey, B.G., et al. (2009) High-density renewable fuels based on the selective dimerization of pinenes. Energy Fuels 24, 267-273

11 Harvey, B.G., et al. (2014) High-density biosynthetic fuels: the intersection of heterogeneous catalysis and metabolic engineering. Phys. Chem. Chem. Phys. 16, 9448-9457

12 Meylemans, H.A., et al. (2014) Low-temperature, solvent-free dehydration of cineoles with heterogeneous acid catalysts for the production of high-density biofuels. $\mathrm{J}$. Chem. Technol. Biotechnol. 89, 957-962 
13 Harvey, B.G., et al. The United States of America as Represented by the Secretary of the Navy, Washington, DC (US). High density renewable fuels based on the selective dimerization of pinenes, US8227651B1

14 Harvey, B.G., et al. The United States of America as Represented by the Secretary of the Navy, Washington, DC (US). Methods for the production of renewable dimethyl JP10, US9327279B2

15 Harvey, B.G., et al. The United States of America as Represented by the Secretary Navy, Washington, DC (US). Efficient conversion of pure and mixed terpene feedstocks to high density fuels, US8975463B1

16 Solomon, S., et al. (2009) Irreversible climate change due to carbon dioxide emissions. Proc. Natl. Acad. Sci. U.S.A. 106, 1704-1709

17 Liu, D., et al. (2016) Advances and perspectives on the use of CRISPR/Cas9 systems in plant genomics research. Curr. Opin. Plant Biol. 30, 70-77

18 Peralta-Yahya, P.P. and Keasling, J.D. (2010) Advanced biofuel production in microbes. Biotechnol. J 5, 147-162

19 Peralta-Yahya, P.P., et al. (2011) Identification and microbial production of a terpene-based advanced biofuel. Nat. Commun. 2, 483

20 Kirby, J., et al. (2014) Enhancing terpene yield from sugars via novel routes to 1deoxy-o-xylulose 5-phosphate. Appl. Environ. Microbiol. 81, 130-138

21 Zhang, H., et al. (2014) Microbial production of sabinene-a new terpene-based precursor of advanced biofuel. Microb Cell Fact 13, 3826-3831

22 Yang, J., et al. (2016) Biosynthesis of $\beta$-caryophyllene, a novel terpene-based highdensity biofuel precursor, using engineered Escherichia coli. Renewable Energy 99, 216-223

23 Yang, J. and Nie, Q. (2016) Engineering Escherichia coli to convert acetic acid to $\beta$ caryophyllene. Microb Cell Fact 15, 1

24 Peralta-Yahya, P.P., et al. (2012) Microbial engineering for the production of advanced biofuels. Nature 488, 320-328

25 Erickson, B. and Winters, P. (2012) Perspective on opportunities in industrial biotechnology in renewable chemicals. Biotechnol. J 7, 176-185 
26 Dunlop, M.J. (2011) Engineering microbes for tolerance to next-generation biofuels. Biotechnology Biofuels 4, 32

27 Arendt, P., et al. (2016) Synthetic biology for production of natural and new-to-nature terpenoids in photosynthetic organisms. Plant J. DOI: 10.1111/tpj.13138

28 Pitera, D.J., et al. (2007) Balancing a heterologous mevalonate pathway for improved isoprenoid production in Escherichia coli. Metab. Eng. 9, 193-207

29 Hemmerlin, A. (2013) Post-translational events and modifications regulating plant enzymes involved in isoprenoid precursor biosynthesis. Plant Sci. 203, 41-54

30 Chang, M.C. and Keasling, J.D. (2006) Production of isoprenoid pharmaceuticals by engineered microbes. Nat. Chem. Biol. 2, 674-681

31 Goodger, J.Q., et al. (2010) Isolation of intact sub-dermal secretory cavities from Eucalyptus. Plant Methods 6, 20

32 King, D.J., et al. (2006) The accumulation of terpenoid oils does not incur a growth cost in Eucalyptus polybractea seedlings. Funct. Plant Biol. 33, 497-505

33 Lange, B.M. and Ahkami, A. (2013) Metabolic engineering of plant monoterpenes, sesquiterpenes and diterpenes-current status and future opportunities. Plant Biotechnol. J. 11, 169-196

34 Lange, B.M., et al. (2011) Improving peppermint essential oil yield and composition by metabolic engineering. Proc. Natl. Acad. Sci. U.S.A. 108, 16944-16949

35 Davies, F.K., et al. (2014) Toward a photosynthetic microbial platform for terpenoid engineering. Photosynthesis Res. 123, 265-284

36 Hinchee, M., et al. (2011) Short-rotation woody crops for bioenergy and biofuels applications. In Biofuels, pp. 139-156, Springer

37 Jansson, C., et al. (2010) Phytosequestration: carbon biosequestration by plants and the prospects of genetic engineering. Bioscience 60, 685-696

38 Johansson, A. (1982) By-product recovery and valorisation in the kraft industry: A review of current trends in the recovery and use of turpentine and tall oil derivatives. Biomass 2, 103-113

39 Tuskan, G. (1998) Short-rotation woody crop supply systems in the United States: what do we know and what do we need to know? Biomass and Bioenerg 14, 307-315 40 Myburg, A.A., et al. (2014) The genome of Eucalyptus grandis. Nature 510, 356-362 
41 Fernando, S.C., et al. (2016) Plant regeneration through indirect organogenesis and genetic transformation of Eucalyptus polybractea RT Baker. Industrial Crops and Products 86, 73-78

42 Külheim, C., et al. (2015) The Eucalyptus terpene synthase gene family. BMC Genomics 16, 450

43 Goss, K., et al. (2014) Sustainable mallee jet fuel: Sustainability and life cycle assessment for supply to Perth airport, western Australia. Future Farm Industries CRC 44 Joyce, B. and Stewart, C. (2012) Designing the perfect plant feedstock for biofuel production: using the whole buffalo to diversify fuels and products. Biotechnol. Adv. 30, 1011-1022

45 Padovan, A., et al. (2014) The evolution of foliar terpene diversity in Myrtaceae. Phytochem. Rev. 13, 695-716

46 King, D.J., et al. (2006) Regulation of oil accumulation in single glands of Eucalyptus polybractea. New Phytol. 172, 440-451

47 Goodger, J.Q. and Woodrow, I.E. (2010) The influence of micropropagation on growth and coppicing ability of Eucalyptus polybractea. Tree Physiol 30, 285-296

48 lqbal, Z., et al. (2011) Variation in composition and yield of foliage oil of Eucalyptus polybractea. J. Chem. Soc. Pak 33, 183-187

$49 \mathrm{Wu}, \mathrm{H}$., et al. (2008) Production of mallee biomass in western Australia: Energy balance analysis. Energy Fuels 22, 190-198

50 Milthorpe, P., et al. (1998) Optimum planting densities for the production of eucalyptus oil from blue mallee (Eucalyptus polybractea) and oil mallee (E. kochii). Ind Crops Prod 8, 219-227

51 Goodger, J.Q., et al. (2007) Examination of the consistency of plant traits driving oil yield and quality in short-rotation coppice cultivation of Eucalyptus polybractea. For. Ecol. Manage. 250, 196-205

52 Gscheidmeier, M. and Fleig, H. (2000) Turpentines. Wiley-VCH Verlag GmbH \& Co. $\mathrm{KGaA}$

53 Yuan, J.S., et al. (2008) Plants to power: Bioenergy to fuel the future. Trends in Plant Sci 13, 421-429 
54 Andrew, R.L., et al. (2013) Intensive sampling identifies previously unknown chemotypes, population divergence and biosynthetic connections among terpenoids in Eucalyptus tricarpa. Phytochemistry 94, 148-158

55 Oates, C.N., et al. (2015) The transcriptome and terpene profile of Eucalyptus grandis reveals mechanisms of defence against the insect pest, Leptocybe invasa. Plant Cell Physiol. 56, 1418-1428

56 Llusià, J. and Peñuelas, J. (2000) Seasonal patterns of terpene content and emission from seven Mediterranean woody species in field conditions. Am. J. Bot. 87, 133-140

57 Zulak, K.G., et al. (2009) Targeted proteomics using selected reaction monitoring reveals the induction of specific terpene synthases in a multi-level study of methyl jasmonate-treated Norway spruce (Picea abies). Plant J. 60, 1015-1030

58 Jones, C.G., et al. (2008) Isolation of cDNAs and functional characterisation of two multi-product terpene synthase enzymes from sandalwood, Santalum album L. Arch. Biochem. Biophys. 477, 121-130

59 Phillips, M.A., et al. (2003) cDNA isolation, functional expression, and

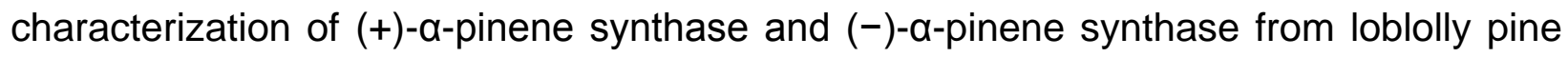
(Pinus taeda): Stereocontrol in pinene biosynthesis. Arch. Biochem. Biophys. 411, 267276

60 Irmisch, S., et al. (2015) One amino acid makes the difference: the formation of entkaurene and 16a-hydroxy-ent-kaurane by diterpene synthases in poplar. BMC Plant Biol. 15, 262

61 Kainer, D., et al. (2015) Genomic approaches to selection in outcrossing perennials: focus on essential oil crops. Theor. Appl. Genet. 128, 2351-2365

62 Kitano, H. (2002) Systems biology: a brief overview. Science 295, 1662-1664

63 Nielsen, J. and Jewett, M.C. (2008) Impact of systems biology on metabolic engineering of Saccharomyces cerevisiae. FEMS Yeast Res. 8, 122-131

64 Champagne, A. and Boutry, M. (2013) Proteomic snapshot of spearmint (Mentha spicata L.) leaf trichomes: a genuine terpenoid factory. Proteomics 13, 3327-3332 65 Voo, S.S., et al. (2012) Assessing the biosynthetic capabilities of secretory glands in citrus peel. Plant Physiol. 159, 81-94 
66 Champagne, A., et al. (2012) In-depth proteome mining of cultured Catharanthus roseus cells identifies candidate proteins involved in the synthesis and transport of secondary metabolites. Proteomics 12, 3536-3547

67 Wang, W., et al. (2009) Global characterization of Artemisia annua glandular trichome transcriptome using 454 pyrosequencing. BMC Genomics 10, 465

68 Hefer, C., et al. (2011) The Eucalyptus genome integrative explorer (EucGenIE): a resource for Eucalyptus genomics and transcriptomics. In BMC Proceedings, pp. 1-1, Springer

69 Pasoreck, E.K., et al. (2016) Terpene metabolic engineering via nuclear or chloroplast genomes profoundly and globally impacts off-target pathways through metabolite signalling. Plant Biotechnol. J.

70 Mizrachi, E. and Myburg, A.A. (2016) Systems genetics of wood formation. Curr. Opin. Plant Biol. 30, 94-100

71 Fukushima, A., et al. (2009) Integrated omics approaches in plant systems biology. Curr. Opin. Chem. Biol. 13, 532-538

72 Otero, J.M., et al. (2013) Industrial systems biology of Saccharomyces cerevisiae enables novel succinic acid cell factory. PloS One 8, e54144

73 Kalachanis, D. and Psaras, G. (2005) Structure and development of the secretory cavities of Myrtus communis leaves. Biol. Plant. 49, 105-110

74 Heskes, A., et al. (2012) Multiphoton fluorescence lifetime imaging shows spatial segregation of secondary metabolites in Eucalyptus secretory cavities. J. Microsc 247, 33-42

75 List, S., et al. (1995) Functional anatomy of the oil glands of Melaleuca alternifolia (Myrtaceae). Aust. J. Bot. 43, 629-641

76 Schaefer, D.W. and Agamalian, M.M. (2004) Ultra-small-angle neutron scattering: a new tool for materials research. Curr. Opin. Solid State Mater. Sci. 8, 39-47

77 Nishiyama, Y., et al. (2014) Structural coarsening of aspen wood by hydrothermal pretreatment monitored by small-and wide-angle scattering of $\mathrm{X}$-rays and neutrons on oriented specimens. Cellulose 21, 1015-1024 
78 Pingali, S.V., et al. (2014) Morphological changes in the cellulose and lignin components of biomass occur at different stages during steam pretreatment. Cellulose 21, 873-878

79 De Paoli, H.C., et al. (2016) An innovative platform for quick and flexible joining of assorted DNA fragments. Sci Rep 6, 19278

80 Yang, F., et al. (2013) Engineering secondary cell wall deposition in plants. Plant Biotechnol. J. 11, 325-335

81 Baltes, N.J. and Voytas, D.F. (2015) Enabling plant synthetic biology through genome engineering. Trends Biotechnol. 33, 120-131

82 Aggarwal, D., et al. (2011) Agrobacterium tumefaciens mediated genetic transformation of selected elite clone (s) of Eucalyptus tereticornis. Acta Physiol Plant 33, 1603-1611

83 Bönisch, F., et al. (2014) A UDP-glucose: monoterpenol glucosyltransferase adds to the chemical diversity of the grapevine metabolome. Plant Physiol. 165, 561-581

84 Luan, F., et al. (2004) Enantioselective analysis of free and glycosidically bound monoterpene polyols in Vitis vinifera L. cvs. Morio Muscat and Muscat Ottonel: evidence for an oxidative monoterpene metabolism in grapes. J. Agric. Food Chem. 52, 20362041

85 Kortbeek, R., et al. (2016) Engineering of tomato glandular trichomes for the production of specialized metabolites. Methods Enzymol.

86 Doran, J., et al. (1998) Variation in first-harvest oil production in Eucalyptus radiata. Australian Forestry 61, 27-33

87 Baker, G., et al. (2014) Highly improved tea tree varieties to maximise profit. Rural Industries Research and Development Corporation 13, RIRDC Project No PRJ - 003689 88 Goodger, J.Q. and Woodrow, I.E. (2012) Genetic determinants of oil yield in Eucalyptus polybractea RT Baker. Trees 26, 1951-1956

89 Wildy, D. and Pate, J. (2002) Quantifying above-and below-ground growth responses of the western Australian oil mallee, Eucalyptus kochii subsp. plenissima, to contrasting decapitation regimes. Ann. Bot. 90, 185-197

90 Zalesny, R.J., et al. (2011) Woody biomass from short rotation energy crops. American Chemical Society: Washington, DC 1067, 27-63 
91 Stanturf, J.A., et al. (2013) Eucalyptus beyond its native range: Environmental issues in exotic bioenergy plantations. International Journal of Forestry Research 2013, 1-5 92 Denny, E. (2003) Distillation of Eucalyptus leaf oils: Theory and practice. CRC Press 93 Jiang, Z., et al. (2016) Extraction and analysis of terpenes/terpenoids. Curr Protoc Plant Biol., 345-358

94 Ormeno, E., et al. (2011) Extracting and trapping biogenic volatile organic compounds stored in plant species. Trends Anal. Chem. 30, 978-989

95 Hellier, P., et al. (2013) The importance of double bond position and cis-trans isomerisation in diesel combustion and emissions. Fuel 105, 477-489

96 Saad, N.Y., et al. (2013) Major bioactivities and mechanism of action of essential oils and their components. Flavour Fragrance J. 28, 269-279

97 Harvey, B.G., et al. (2015) High-density renewable diesel and jet fuels prepared from multicyclic sesquiterpanes and a 1-hexene-derived synthetic paraffinic kerosene. Energy Fuels 29, 2431-2436

98 Beller, H.R., et al. (2015) Natural products as biofuels and bio-based chemicals: fatty acids and isoprenoids. Nat. Prod. Rep. 32, 1508-1526

99 Peralta-Yahya, P.P., et al. (2011) Identification and microbial production of a terpene-based advanced biofuel. Nature Communications 2, 483

100 Coppen, J.J. (2003) Eucalyptus: the genus Eucalyptus. CRC Press

101 Brophy, J.J. and Southwell, I.A. (2002) Eucalyptus chemistry. Eucalyptus: the genus Eucalyptus, 102

102 Elaissi, A., et al. (2007) Contribution to the qualitative and quantitative study of seven Eucalyptus species essential oil harvested of Hajeb's Layoun arboreta (Tunisia). J Essent Oil Bear PI 10, 15-25

103 Bignell, C., et al. (1998) Volatile leaf oils of some south-western and southern Australian species of the genus Eucalyptus. Flavour Fragrance J. 13, 43-47

104 Vranová, E., et al. (2013) Network analysis of the MVA and MEP pathways for isoprenoid synthesis. Annu. Rev. Plant Biol. 64, 665-700 
Table 1. Specific terpenes identified that are a suitable replacement or blend-in for current petroleum-derived fuels and comparison with known fuels. Density values were extracted from the cited literature and is based on the conditions outlined therein.

\begin{tabular}{|c|c|c|c|}
\hline Compound & Chemical structure & $\begin{array}{l}\text { Density } \\
\left(\mathrm{g} \mathrm{ml}^{-1}\right)\end{array}$ & References \\
\hline JP-10 & & 0.94 & [6] \\
\hline RJ-5 & & 1.08 & {$[6]$} \\
\hline Gasoline & & 0.74 & [6] \\
\hline Biodiesel & & 0.88 & [6] \\
\hline Bisabolene & & 0.82 & [19] \\
\hline Camphene & & 0.84 & [6] \\
\hline Caryophyllene & & 0.90 & [97] \\
\hline 1,8-Cineole & & 1.01 & {$[12,96]$} \\
\hline
\end{tabular}




\begin{tabular}{|c|c|c|}
\hline$(-)-\beta$-Citronellene & 0.76 & [8] \\
\hline & $0.84-0.88$ & [8] \\
\hline & 0.89 & [8] \\
\hline & 0.88 & [96] \\
\hline & 0.92 & [8] \\
\hline Limonene & 0.85 & [3] \\
\hline Linalool & 0.87 & [8] \\
\hline a-Pinene & 0.86 & {$[6,10]$} \\
\hline$\beta$-Pinene & 0.86 & {$[6,10]$} \\
\hline
\end{tabular}




Sabinene


Table 2. List of candidate species for production of high energy terpenes in Eucalyptus.

\begin{tabular}{|c|c|c|c|c|c|}
\hline Species & $\begin{array}{c}\text { Terpene } \\
\text { yield }\end{array}$ & $\begin{array}{c}\text { Geographic } \\
\text { range }\end{array}$ & $\begin{array}{c}\text { Climate } \\
\text { classification }\end{array}$ & $\begin{array}{c}\text { Dominant } \\
\text { terpene }\end{array}$ & References \\
\hline E. eremicola & $3.1 \% \mathrm{DM}$ & Cent WA, SA & $\begin{array}{l}\text { BWh, BWk, } \\
\text { BSh, BSk }\end{array}$ & $\beta$-pinene & {$[100,101]$} \\
\hline E. eremophila & $\begin{array}{c}1.8-5.2 \% \\
D M\end{array}$ & S WA & BSh, BSk, Csa & a-pinene & [100-102] \\
\hline E. forrestiana & $3.3 \% \mathrm{DM}$ & SWA & BSk, Csb & a-pinene & {$[100,101]$} \\
\hline E. macrocarpa & $2.5 \% \mathrm{DM}$ & W WA & Csa & a-pinene & {$[100,101]$} \\
\hline E. pimpiniana & $1.9 \% \mathrm{DM}$ & $\begin{array}{c}\text { S Cent SA, } \\
\text { WA }\end{array}$ & BWh & a-pinene & {$[100,101]$} \\
\hline & $0.6-1.2 \%$ & & & & \\
\hline E. platypus & FW & SWA & BSk, Csb & $\alpha$-pinene & {$[100,101]$} \\
\hline E. suggrandis & $1.1 \% \mathrm{DM}$ & SW WA & BSk, Csb & a-pinene & {$[100,103]$} \\
\hline E. striaticalyx & $\begin{array}{c}0.7-4.4 \% \\
\text { DM }\end{array}$ & $\begin{array}{l}\text { Cent WA, } \\
\text { SW SA }\end{array}$ & BWh & a-pinene & {$[100,101]$} \\
\hline & $1.3-4.0 \%$ & & & & \\
\hline E. camphora & DM & SE Aus & Cfa, Cfb & a-pinene & {$[100,101]$} \\
\hline E. morrisii & $1.7 \% \mathrm{FW}$ & Cent NSW & BSh, BSk, Cfa & a-pinene & {$[100,101]$} \\
\hline$E$. & & & BWh, BSh, & & \\
\hline ebbanoensis & $1.8 \% \mathrm{DM}$ & W, Cent WA & Csa & a-pinene & {$[100,103]$} \\
\hline E. longicornis & $1.2 \% \mathrm{FW}$ & SW WA & $\begin{array}{c}\text { BSk, CSb, } \\
\text { Csa, BSh, } \\
\text { BWh }\end{array}$ & $\alpha$-pinene & {$[100,101]$} \\
\hline E. bosistoana & $1-1.5 \%$ & SE NSW, Vic & Cfb, Cfa & a-pinene & {$[100,101]$} \\
\hline
\end{tabular}




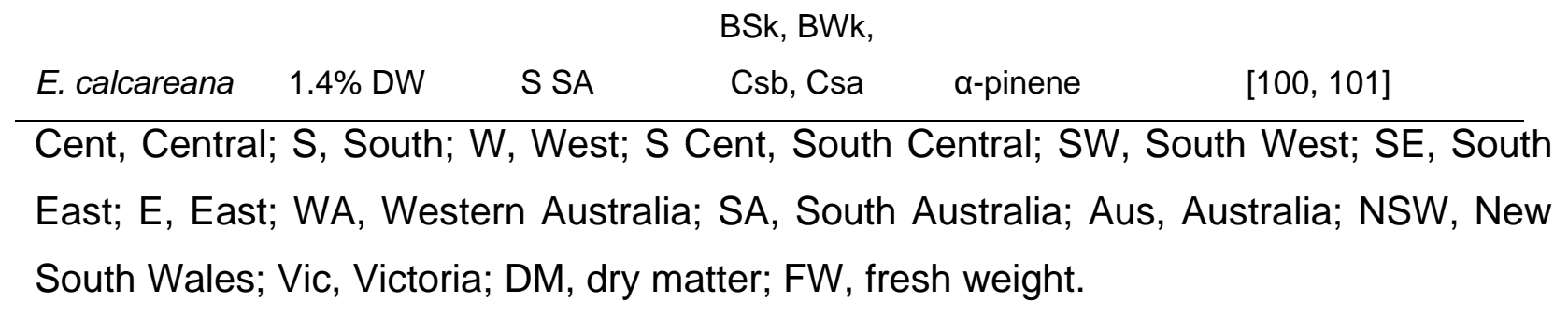


Figure 1. Expression profiles of putative functional homologs of the terpene precursor mevalonate (MVA) and methyl-D-erythritol 4-phosphate (MEP) pathway in Eucalyptus grandis. Pathway was adapted from Vranová et al. [104], and putative Eucalyptus orthologs are based on the expect-value ( $E$-value) of sequence alignment $\leq$ 1.0E-10. Expression data was extracted from EucGenIE [68] and is expressed as fragments per kilobase of transcript per million mapped reads (FPKM). Data is ordered as follows: $Y L$, young leaf; ML, mature leaf; ST, shoot tips; F, flowers; R, roots; Ph, phloem and IX, immature xylem with yellow indicating lowest expression and red the highest expression between tissue. Enzyme abbreviated names: AACT, acetyl-CoA $C$ acetyltransferase; HMGS, 3-hydroxy-3-methylglutaryl-CoA synthase; HMGR, 3-hydroxy3-methylglutaryl-CoA reductase; MK, mevalonate kinase; PMK, phospho-MVA kinase; MPDC, diphospho-MVA decarboxylase; IPPI, isopentenyl diphosphate $\Delta$-isomerase; DXS, 1-deoxy-D-xylulose 5-phosphate synthase; DXR, 1-deoxy-D-xylulose 5-phosphate reductoisomerase; MCT, 2-C-methyl-D-erythritol 4-phosphate cytidylyltransferase; CMK, 4-(cytidine 5'-diphosphate)-2-C-methyl-D-erythritol kinase; MDS, 2-C-methyl-Derythritol 2,4-cyclodiphosphate synthase; HDS, 4-hydroxy-3-methylbut-2-enyldiphosphate synthase; HDR, 4-hydroxy-3-methylbut-2-enyl-diphosphate reductase. 
Figure 2. Gaps in our understanding of proteins involved in the biosynthesis of specific terpenes. User-designed plants for biofuel terpenes, using synthetic biology, will require a comprehensive inventory of proteins specific to this process. Examples of these unknown proteins (represented by question marks) include signaling or receptor proteins such as kinases that elicit specific terpene biosynthesis, preferentially expressed enzyme variants, transporter proteins for precursor metabolites (from the mesophyll to the secretory cells) and secondary metabolites (from the secretory cells to the storage cavity), and regulatory proteins such as transcription factors. While not illustrated, modifying proteins such as glycosyltransferases, which increases the stability of terpenes within the storage cavities at ambient temperatures, is also an important consideration. 


\section{Figure 3. Proposed roadmap for commercialization of specific terpenes from}

plants. We propose a system biology approach using omics profiling of different plant organs such as oil glands (Strategy 1) or across developmental stages (Strategy 2). Generated omics data, including transcriptome, proteome, and metabolome profiling, will be integrated using appropriate mathematical and statistical models to produce a candidate gene list and reveal co-expression patterning across tissues and gene-gene correlations within networks. This information will be used for strategic metabolic engineering via synthetic biology or selective breeding strategies to customize plants for specific terpenes. In combination with established silviculture techniques for maintaining elite lines, and optimized extraction protocols to minimize terpene lost during harvesting, will fortify these tailored plants as a viable source of terpenes for specialty biofuels. 


\section{Box 1. Plant Terpene Biosynthetic Pathway.}

Terpenes, terpenoids, and isoprenoids are derived from the $\mathrm{C}_{5}$ building blocks, isopentenyl diphosphate (IPP), and the IPP isomer, dimethylallyl diphosphate (DMAPP) via two compartmentalized pathways: the cytoplasmic/ER-localized mevalonic acid (MVA) pathway and the plastidial methyl-D-erythritol 4-phosphate (MEP) pathway. IPP and DMAPP are synthesized from acetyl-CoA in the MVA pathway and from pyruvate with glyceraldehyde 3-phosphate in the MEP pathway. Prenyltransferases catalyze the head-to-tail condensation reaction of IPP and DMAPP to generate prenyl pyrophosphate molecules varying in their five-carbon units, including $\mathrm{C}_{10}$ geranyl pyrophosphate (GPP), $\mathrm{C}_{15}$ farnesyl pyrophosphate (FPP), and $\mathrm{C}_{20}$ geranylgeranyl pyrophosphate (GGPP). These prenyl pyrophosphate substrates are used to synthesize cyclic/acyclic mono-, sesqui-, and diterpenes by a midsize family of genes, the terpene synthases (TPSs). Generally, mono- and diterpenes are synthesized from the precursors produced in the MEP pathway, while sesquiterpenes are synthesized from the precursors produced in the MVA pathway; however, variations do occur. These terpenes can undergo further modifications for function including (de)esterification, (de)methylation, glycosylation, isomerization, oxidation, reduction and/or decorations with functional groups to the terpene skeleton, thereby increasing the terpene diversity to more than 40,000 reported structures. 


\section{Glossary}

Metabolic engineering: targeted rewiring of the metabolic network of an organism, using genetic modifications, to optimally produce target metabolites and/or decrease undesirable products.

Selective breeding: a process of developing a desired phenotype by pre-selecting specific individuals that will interbreed. Also referred to as artificial selection.

Silviculture: the science of forest management, particularly in controlling the establishment, maintenance, and health of the forest for a desired and sustainable need. Silviculture techniques are typically based on observation and practice that allows the development of modified practices for new applications.

Specialty biofuels: molecules or compounds beyond ethanol that have the potential to serve as biofuels. These compounds can be used as standalone fuels or blended with current petroleum-derived fuels.

Synthetic biology: a rational and systematic construction of new biological machineries or redesign of the existing biological systems via genetic modifications for specific needs or products.

Systems biology: a study of the complex biological system integrating measured phenotypes (omics-level data) using mathematical and/or probabilistic modeling to understand and predict the emergent phenotype of an organism.

Terpene content: here, both composition and concentration of terpenes within a single species. 


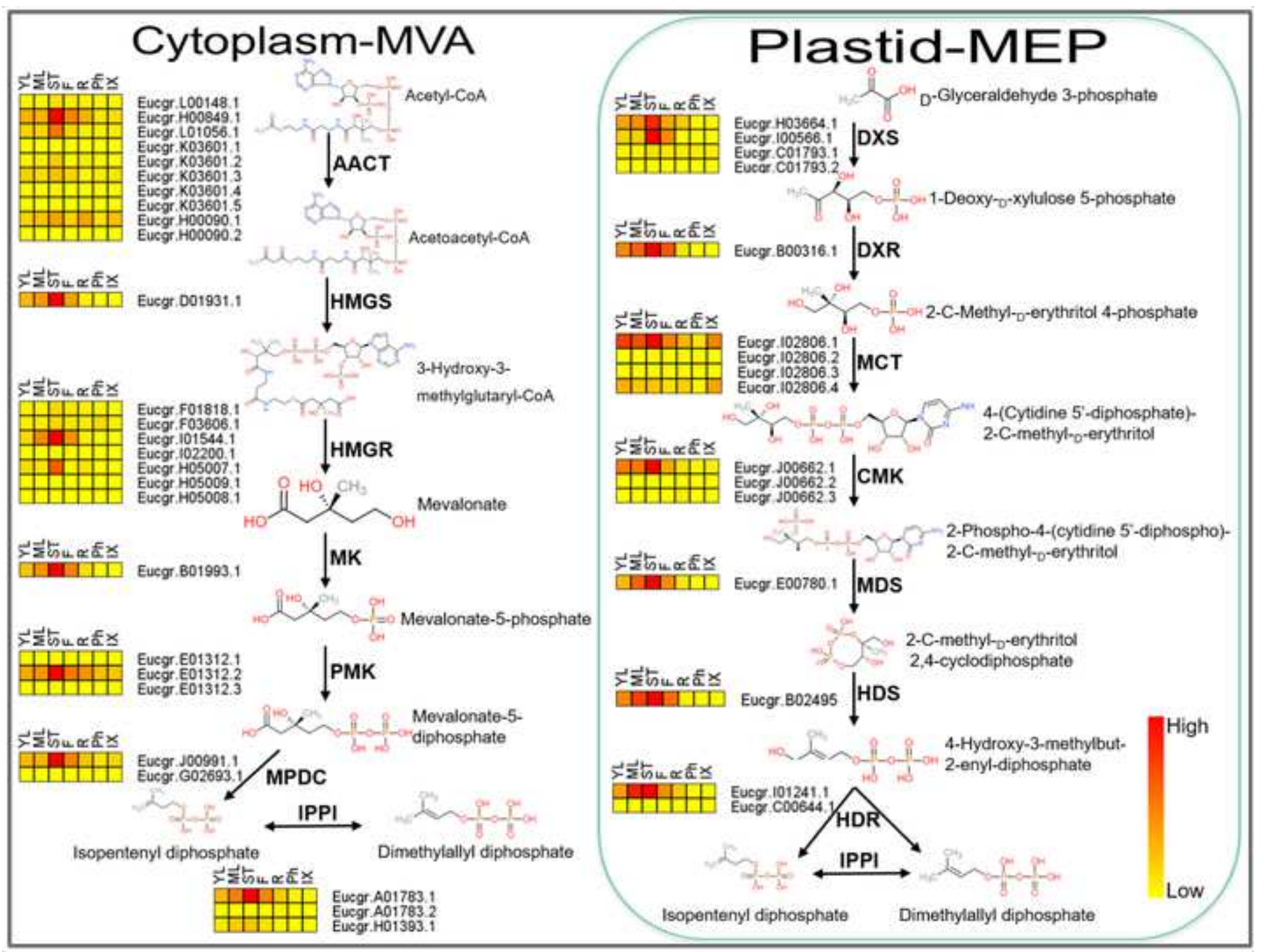

\section{Cytoplasm-MVA}

icor $H 00849$

Euegr. Lo1056.

sgr. Ko3601.

$\mathrm{K} 03601$

Egr. K03601.

Eucgr Hoougo.



비밀 Eucgr D01931.1

HMGS

3-Hydroxy-3-

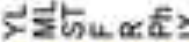

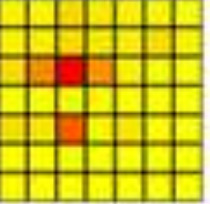

Eucgr F01818, Eucgr. $F 03606.9$

er 102200

uegr 705009

Hor

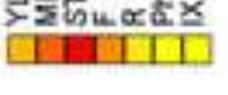


Eucgr.B02495 $\downarrow$ HDS

as 4 -Hydroxy-3-methylbut-

Isopentenyl diphosphate

Diphosphate 


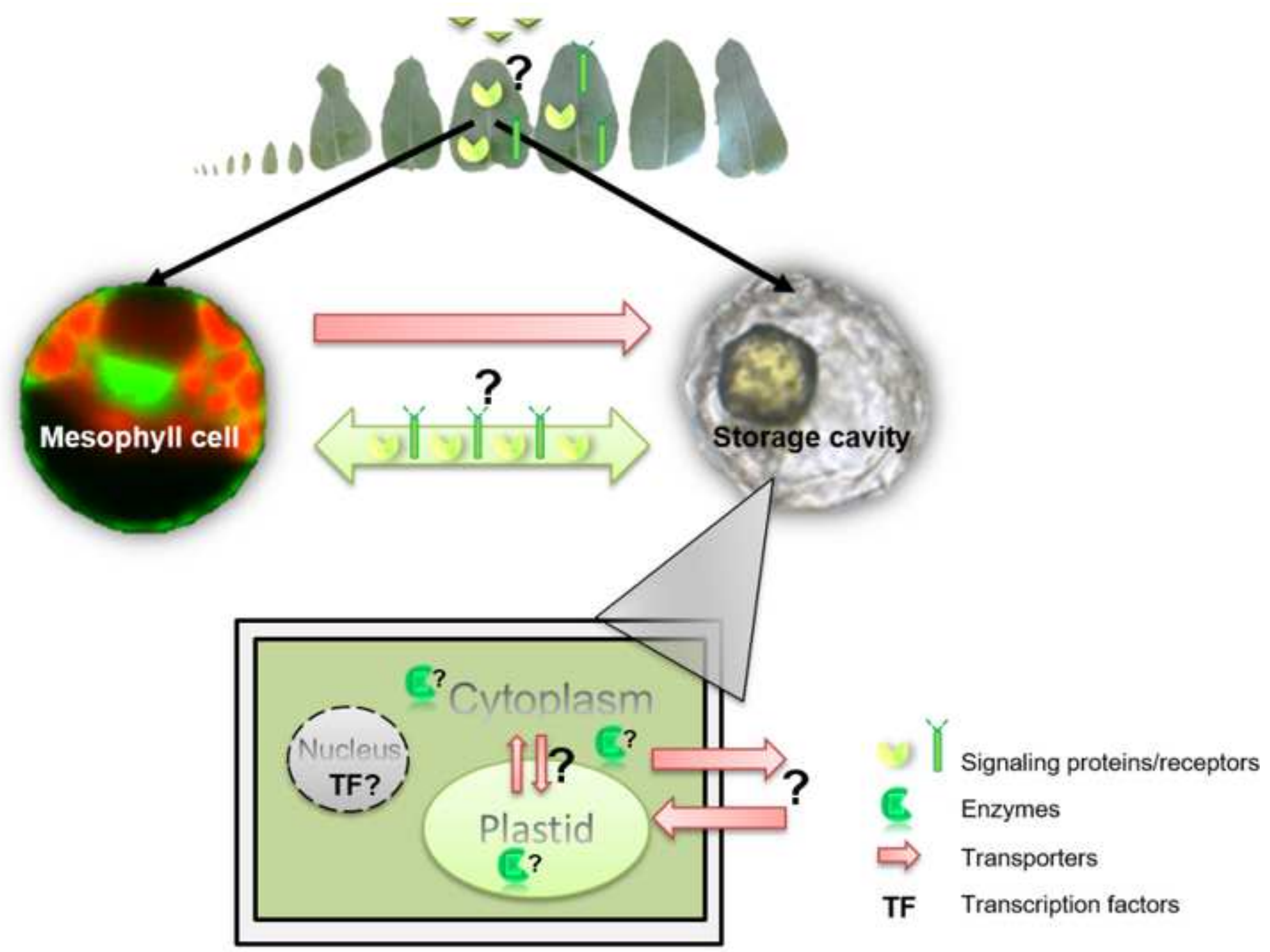




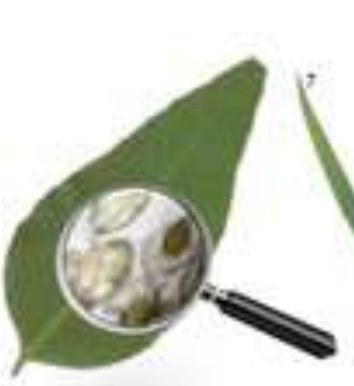

Strategy 1

Strategy 2

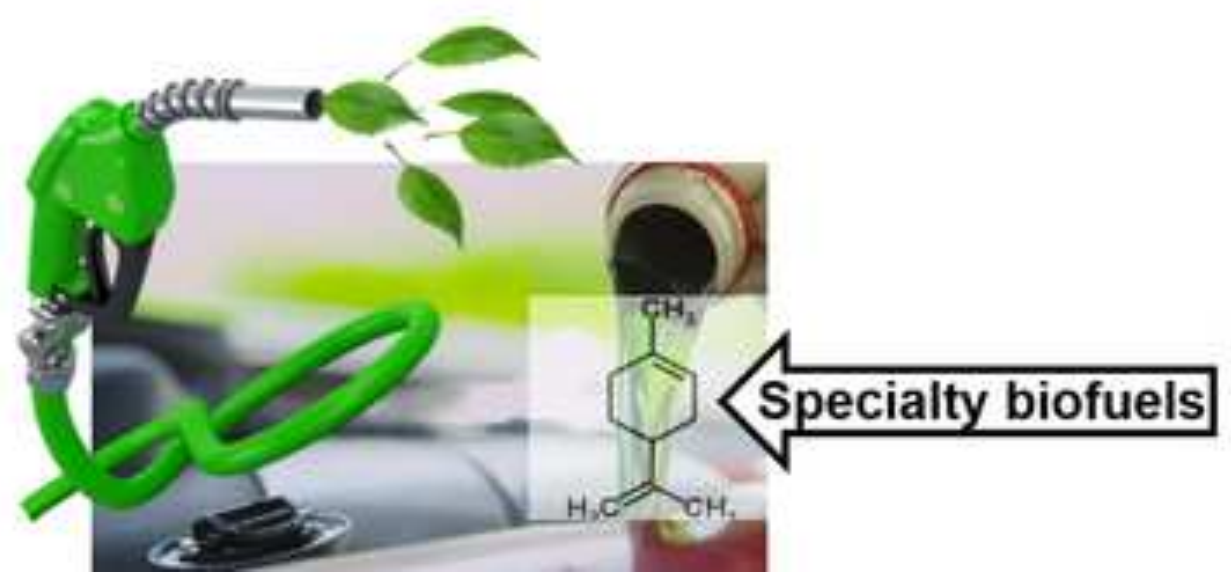

\section{Omics profiling}

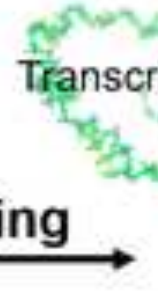

Candidate genes



Co-expression

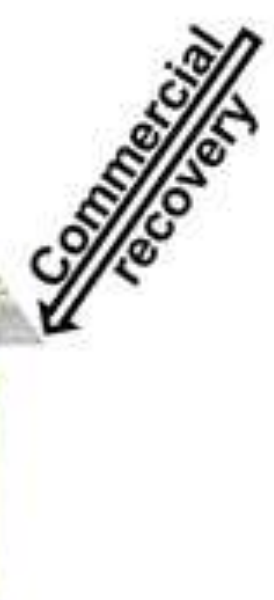

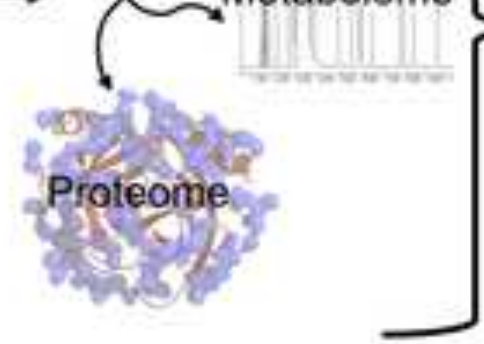

integration

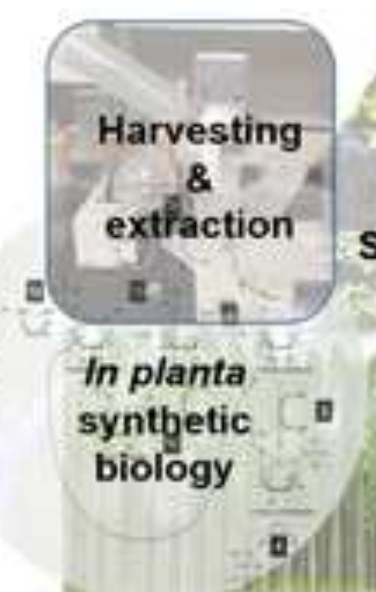

Selective breeding 